\title{
The ICOM 3.0 Intelligent Conceptual Modelling tool and methodology
}

\author{
Pablo R. Fillottrani ${ }^{1}$, Enrico Franconi ${ }^{2}$, and Sergio Tessaris ${ }^{2}$ \\ 1 Universidad Nacional del Sur, and Comisión de Investigaciones Científicas de la provincia de \\ Buenos Aires, Argentina

\section{Introduction}

ICOM is an advanced conceptual modelling tool, which allows the user to design multiple ER or UML class diagrams with inter- and intra-model constraints. Complete logical reasoning is employed by the tool to verify the specification, infer implicit facts, devise stricter constraints, and manifest any inconsistency.

For the ontology creation and maintenance tasks, ICOM interface supports ontology engineers in engineering ontologies that meets clear and measurable quality criteria. Indeed, recently we observe the development of large numbers of ontologies which have, however, usually been developed in an ad hoc manner by domain experts, often with only a limited understanding of the semantics of ontology languages. The result is that many ontologies are of low quality - they make poor use of the languages in which they are written and do not accurately capture the author's rich knowledge of the domain. This problem becomes even more acute as ontologies are maintained and extended over time, often by multiple authors. Poor quality ontologies usually require localised "tuning" in order to achieve the desired results within applications. This leads to further degradation in their overall quality, increases the brittleness of the applications that use them, and makes interoperability and reuse difficult or impossible. To overcome these problems tools are needed which support the design and the development of the basic infrastructure for building, merging, and maintaining ontologies.

The leverage of automated reasoning to support the domain modelling is enabled by a precise semantic definition of all the elements of the class diagrams. The diagrams and inter-model constraints are internally translated into a class- based logic formalism. The same underlying logic enables the use of a view definition language to specify additional constraints, not captured at the diagram level. The conceptual modelling language supported by ICOM can express:

- the standard Extended Entity-Relationship data model or the standard UML class diagrams (we are working on providing Object-Role modelling ORM [5] as well), enriched with disjoint and covering constraints and definitions attached to classes and relations by means of view expressions over other classes and relationships in the ontology; 
- inter-ontology mappings, as inclusion and equivalence statements between view expressions involving classes and relationships possibly belonging to different ontologies.

The tool allows for the creation, the editing, the managing, and the storing of several interconnected ontologies, with a user friendly graphical interface. The main purpose of the ICOM project is not to provide to the ontology community a robust tool potentially replacing the many other tools available; we do not claim that ICOM is currently more usable than any of the existing conceptual modelling tools for ontology design (such as, for example, [8, 1]). ICOM is meant to be a proof of concept, willing to showcase two main points:

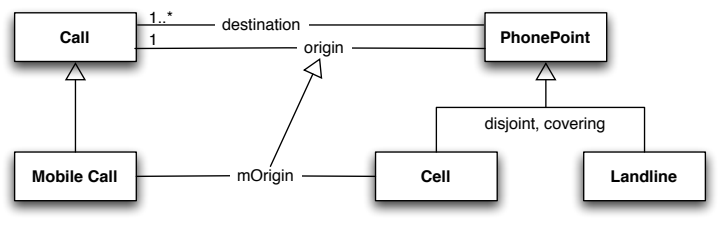

(a) Without deductions

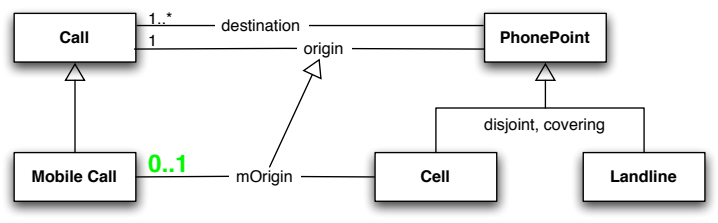

(b) With deductions

Fig. 1: First ontology design scenario.

1. the effectiveness of using a class diagram graphical syntax for expressing ontologies, even with complex languages;

2. the emphasis to the use of complex automated reasoning tasks to deduce implied facts, as opposed to mere subsumption (classification) and consistency.

The two above points are novel and in our opinion very important in the context of the existing ontology design tools and methodologies (see next Section). Indeed ICOM proves (point 1 ) the feasibility and the ease of use of a class diagram graphical syntax for expressing ontologies, even with complex ontology languages, by relying on the notion of views (which roughly correspond to OCL constructs) in order to capture the (typically very few) cases where a larger expressivity than graphical class diagrams is needed.

ICOM is based on a deduction-complete notion of reasoning support relative to the class diagram graphical syntax (point 2). Users will see the original ontology graphically completed with all the deductions making sense given the provided ontology, and 
expressed in the graphical class diagram language itself. This includes checking class and relationship consistency, discovering implied class and relationship inter-relations (e.g., subsumption) or cardinality constraints, and in general discovering any implied but originally implicit class diagram graphical construct. Customarily, ontology design tools just provide a support limited to class subsumption and consistency.

ICOM provides a general framework to support the typical tasks involved in such activities:

- Authoring of concept descriptions: in this task the author wants to add a new concept description to the ontology or modify a concept description that was already contained in the ontology. This may happen either in the design phase of the ontology or during the maintenance phase. After producing a candidate description of the concept, the author needs to understand the implicit consequences of his modelling and the interaction of this description with the other descriptions in the ontology.

- Structuring of the ontology: in this task the ontology designer wants to improve the structure of an ontology by inserting intermediate concepts into the ontology diagram. He needs support to decide where to add such concepts and how to describe them.

ICOM is a fairly mature project, its first release has been published in 2000 (see [7. 4]). The version 3.0 of the ICOM tool is loosely based on the ICOM tool previously released in 2000 as an Entity-Relationship editor (which had around 3,000 registered installations, mostly in academic environments and for teaching purposes in industry), and a demo of a preliminary version was presented few years ago [3]. The foundations of the user-computer interaction have been radically changed according to the experience of the first ICOM and the research in this last decade. The system has been completely re-implemented, using different graphic libraries. The graphical interface has been completely rewritten to improve the usability and intuitiveness of the tool. Interoperability with other tools is a crucial aspect; so, import and export modules have been developed for XMI 2.x and Description Logics based ontology languages via DIG.

The ICOM tool is written in standard Java 5.0, and it is distributed on Linux, Mac, and Windows machines. ICOM communicates via the DIG 1.1 protocol with a description logic server, such as, for example, RACER. ICOM provides an interface for importing and exporting ontologies in UML-XMI class diagrams format.

ICOM is available as a free download at

http://www.inf.unibz.it/ franconi/icom/

\section{The Ontology Editor}

The Ontology Editor works on projects, which may contain one or more UML class diagrams. The diagrams are referred as models. Multiple projects can be opened at the same time, but objects cannot be moved across them. Only one project is visible at a time and the editing of each project is independent. The user can switch between different projects using the tabs at the top of the project area. 


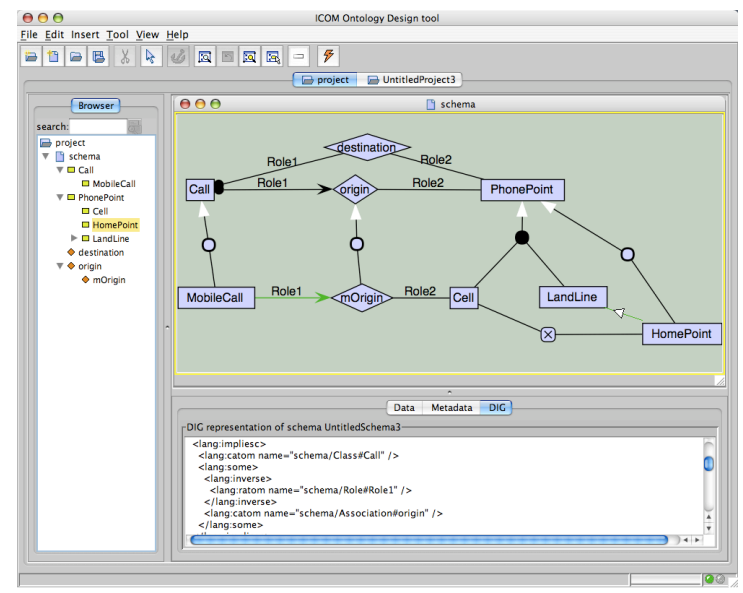

Fig. 2: The ontology design scenario in ICOM.

Figure 2 shows the main window of the Ontology Editor editing a single model. Classes are represented by boxes and n-ary associations by diamonds. Associations may have so-called association classes specifying their attributes. IsA relationships are represented as arrows with a disc in the middle (e.g. see MobileCall and Call).

The tool does not implement special visual techniques for handling very large ontologies. The tasks that it supports, i.e. authoring of concept description and structuring the ontology, are not aimed at working simultaneously with thousands of concepts. However, a set functionalities that are very useful in managing such ontologies are available. First, the interface is zoomable, that is, the level of detail and size of the icons that represent the model can be smoothly changed by pressing the right mouse button and dragging left to zoom our or right to zoom in. Also, the window can be panned by pressing the middle button and dragging. This allows the user to focus the attention in a specific region of the ontology. There are also two dedicated buttons for zooming: one will show the complete graph, and the other will zoom in to show the selected elements. Selection works by left-clicking on icons or by left click and drag; also, there is a button for expanding the selection to all connected nodes, which is very useful in combination with the zoom-to-selection button. Finally, custom automatic layout algorithms for ontologies are under development. These combine known layout algorithms for drawing large graphs, with special conventions used in ontologies, like IsAs hierarchies are drawn top-down and associations are drawn in the middle of related concepts. New metrics to measure the "quality" of ontology graphs were developed with this purpose.

\subsection{Editing Models}

Most of the model editing is done in the project panel, where each model in the project is displayed in a separate model panel. In addition, two dialogues are used to elicit 
additional information about model objects. The attribute domain dialogue allows the domain of attributes to be set. The definition dialogue enables the characterisation of a class or association by means of a view written in the language described in the next Section.

Objects can be created by selecting the appropriate button in the toolbar, or an entry under the Diagram menu, or a contextual menu in the project area. Most objectcreating operations require further inputs to complete the operation. Usually, the user is requested to select an existing object in the diagram (e.g. during the creation of an IsA relationship). In this case, the system will highlight only objects in the diagram suitable for the specific operation.

All the objects of the diagram have a name. Upon their creation the system allocates a new fresh name, which can be edited by the user. To improve the identification of the nodes, when icons become smoller because of the zoom level, all the nodes show their name on a tool-tip when the mouse is hovering over them. Names are scoped by the model they belong; e.g. classes with the same name in different models are considered different.

Metadata fields can be associated to every kind of objects. These fields are ignored in the reasoning process.

The creation of a new class adds a new box in the diagram with a new default name. Every class can optionally have attributes. Attributes are added and edited by means of a specific attribute dialogue. Similar to classes, attributes of the same name in different models are considered different. Attributes of the same name within the same model represent the same attribute. For each attribute, a domain should be indicated. There the set of possible domains is not predefined, and the user is allowed to enter an arbitrary name. Unlike the classes and associations, domains have a global context. Therefore, domains of the same name in different models are considered be the same.

Associations are created by default with no roles. $\mathrm{N}$-ary associations can be specified by adding new roles to existing ones. The creation of a new association introduces a corresponding association class; which can be edited as a normal class (e.g. it can have attributes).

Adding new roles to an existing association requires the user to select the association and a class which restricts the domain of the argument of the association corresponding to the role. Similar to class and associations names, role names have a model scope.

For example, assume there are two models $\mathrm{M} 1$ and $\mathrm{M} 2$, each one with a binary association lives having the roles subject and object. Note that, being association scoped over models, from the global perspective there are two associations M1: l i ves and $\mathrm{M} 2$ : lives. Now, the modelling of the domain requires that $\mathrm{M} 2$ : lives is more specific (i.e. a subset) of M1:lives. Since also role names are scoped over each model, overall there are four different roles. Therefore, the more specific association (M2: lives) inherits the roles of the general one, ending up being of arity four (namely the roles $\mathrm{M} 2$ : ob ject, $\mathrm{M} 2$ : sub ject and M1 : ob ject, $\mathrm{M} 1$ : sub ject).

Roles denote the connection of a class to an association and it is also used to express the cardinality constraints of a class in an association. A role may have two constraints: totality, or the minimum cardinality, and uniqueness representing the maximum cardinality. In the current version of the system, the numbers expressing cardinality are 


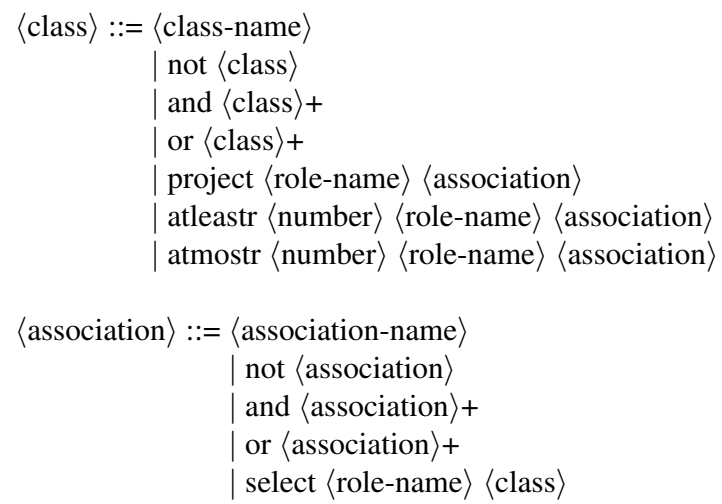

Fig. 3: View definition language

restricted to be 0 and 1 . A minimum cardinality of 1 indicates that all instances of a class must participate in the association at least once (i.e. mandatory constraint). A maximum cardinality of 1 indicates that all instances of a class can only participate once in the association (i.e. functional constraint).

Within a project, equivalence and subset role mappings can be defined between roles in the same or different models. These allow a better characterisation of the relationship between associations across different models. In the former example, M2: lives can be set as a binary association by saying that $\mathrm{M} 1: \mathrm{ob}$ ject contains $\mathrm{M} 2 \mathrm{:object}$, and that $\mathrm{M} 1$ : sub ject contains $\mathrm{M} 2$ : sub ject.

The system enables the user to specify inheritance relationships among classes and associations. The relationships can be arbitrary (e.g. cycles are allowed) provided that classes can only inherit from classes, and associations from associations. Formally, the inheritance is expressed by the inclusion (subclass) constraint.

On the diagram, inheritance is specified by means of IsA links (in the diagram indicated by arrows with a circle in the middle) connecting nodes. IsA links can be specified one-to-one, or many-to-one. The latter groups together more than one (association) class and restrict all of them to be a subclass of the link target.

The possibility of grouping more than one descendant, not only provides way of visually organising the layout of the model; but enable the user to specify additional constraints among the (association) classes. In particular, the covering and disjointness constraints. The first one expresses the fact that the (association) class is equivalent to the union of the specified descendant; while the second constraints the grouped (association) classes to be mutually disjoint.

Note that disjointness among classes is not assumed by default; so, in absence of a specific constraint, (association) classes may overlap. 


\subsection{Inter-Model Axioms}

Additional constraints among classes and associations can be expressed by means of intra- as well as inter-model axioms. The Ontology Editor provides four types of axioms: Node Definition, Equivalence, Subsumption and Disjointness. As discussed in Section 1 these constraints provide a powerful modelling tool in the context of data integration and ontology mapping.

Each class and association can be fully defined by means of a view expression. The view expression language is more expressive that the diagrammatic definition language, so enables the expert user to add constraints that cannot be expressed by the UML diagram alone.

The adopted view language (see Figure 3 ) based on the DLR description logic. A definition has a global context, meaning it can express inter-model relationships as well as intra-model relationships. The view language includes two syntactic sorts: one for classes and one for associations. Full boolean operators are allowed, plus a selection operator (selecting tuples in an association with a specific class type in some named role argument) and a unary projection operator (projecting an association over a named role argument). A generalised projection operator with cardinality restrictions is available as well.

Since a definition can refer to objects in different models, a name-prefix is used in definitions to distinguish objects with the same name but from different models. The name-prefix used is the model's name followed by a colon symbol. For example, class 1 in Model1 and class 1 in Mode 12 would be referred to as Model1:class 1 and Model2: class1 respectively.

Any two (associations) classes in any model can be related by semantic relationships stating their equivalence, subsumption, or disjointness. Creating one of these relationships requires the user to specify source and target node. The system prevents the creation of a relationship between non-homogeneous nodes by restricting the scope of the second node to be selected.

\subsection{Exporting and Importing Projects}

ICOM projects can saved and retrieved in an own XML format, preserving the meaning of all elements including view definitions. It is also possible to import UML class diagrams saved in the XMI format. The tool only recognises the subset of XMI determined by classes, associations, attributes, roles and primitive datatypes defined within an UML model. Functional and mandatory constraints on roles are the only type of imported constraints. Aggregation relationships in the UML model are ignored. We are currently working on exporting projects in XMI files, but this translation would be necessarily carried out with some loss of meaning because, for example, not all view definitions can be expressed in XMI even with attaching OCL expressions to the model elements.

\section{Automated Reasoning}

Although the Ontology Editor can be used as a standalone modelling tool, exploiting its full capabilities requires the coupling of the system with a Description Logic rea- 
soner. Without such an automated reasoning tool the Ontology Editor would be unable to perform deduction-complete automated reasoning over the models. As we noted, this includes checking class and relationship consistency, discovering implied class and relationship inter-relations (e.g., subsumption) or cardinality constraints, and in general discovering any implied but originally implicit class diagram graphical construct.

Instead of implementing its own dedicated reasoner, the Ontology Tool can exploit any DIG enabled DL reasoner (see [2]). Being DIG a standardised communication protocol, the user can choose the most suitable DL reasoner (e.g. the one used by other in-house project), or upgrade to the latest version of the preferred reasoner without being forced to upgrade to a different version of the Ontology Editor.

We adopted the DIG protocol rather than the OWL API [6] because it is language independent rather than based on a Java model. Currently we are in the process of evaluating the migration to OWLLink [9], which is the successor of DIG protocol for the latest OLW 2 standard.

To enable the automated deduction mechanism, the user must specify the URL of the server where the DIG reasoner is available. Note that it is not required that the DIG server should be installed in the same computer as the Ontology Editor. Once the URL to the DIG server has been provided, the system checks the connection to the reasoner to verify its availability. Upon success, the menu item and toolbar button enabling the automated deduction will be enabled.

The so called verification process can be computationally expensive, so it is activated only on user's request. This process includes the following operations. The selected project is encoded into an appropriate Description Logics knowledge base and shipped to the DIG reasoner. Each class, association in the project is checked for satisfiability (i.e. non-emptiness). For each class, association in the project, its equivalent peers, and super-classes are determined. For each class-role-association triple, the system calculates the stricter minimum and maximum cardinality constraints. To perform these operations, the system formulates a sequence of queries to be sent to the DIG reasoner. Accordingly to the received answers the Ontology Editor infers properties of the models in the project. To perform these operations, the system formulates a sequence of queries to be sent to the DIG reasoner, which is linear in the number of project elements. Accordingly to the received answers the Ontology Editor infers properties of the models in the project. The algorithm for this inference is quadratic in the number of concepts and roles, and linear in the number of axioms and IsA links. Thus, the tool can reasonable manage projects with several hundreds of elements, calling a current state-of- the-art reasoner.

After the verification process, the system provides the user with a visual account of the deductions by modifying the appearance of the model diagrams in the project. All unsatisfiable objects will appear in red in the model diagrams. An object is unsatisfiable when necessarily describes an empty set of tuples of objects. Additional non explicit deductions will appear in green, to be distinguished from the user specified elements of the diagrams. Semantically equivalent objects are connected with newly inserted equivalent axiom links. Objects discovered to hold an inclusion relationships between them are connected with subsumption axiom links. Cardinality constraints of roles for that are more restrictive than those originally specified. Although the deductions are 
displayed on the actual diagrams, it is up to the user to decide whether they should be permanently added to the models or discarded. The rational behind this behaviour is that the automated reasoning process may detect unwanted deductions caused by a wrong modelling of the domain. In this case the user should correct the project before any subsequent editing. Another reason is that, in spite of the fact that only the nontrivial deductions are presented, the user is satisfied by the fact that they are implicit without the need of having them explicitly asserted.

The user can discard the deductions and the entire project will be returned to its original state (and any information about unsatisfiability will be discarded). Editing one of the models in the project will also discard the deductions before the editing is carried out. Alternatively, the equivalence, subsumption association, and role cardinality deductions can be added permanently to the project by committing them.

\section{Conclusions and Future Works}

In this paper we presented ICOM, an advanced conceptual modelling tool grounded on more than ten years of research on the use of automated reasoning to support the development and integration of ontologies. ICOM employes a diagrammatic based language to represent most of the constructs used in ontology design; although it enables the use of non graphical ontology languages, experience with users demonstrates that the design of the diagrammatic language is sufficiently expressive to describe rich domains. Moreover, deductions are expressed within the same diagrammatic language; providing a uniform view over design and analysis of models.

By means of use cases we demonstrated the importance of exploiting basic reasoning tasks (as subsumption) in order to provide richer information on ontologies. This is a crucial step towards guaranteeing the quality of the ontologies designed using a tool like ICOM.

The research and development of ICOM continues on two main tracks: from one side we are improving the modelling workflow by considering alternative modelling languages and reasoning services; while on the other hand we are enhancing the user experience by improving the graphical user interface and the interoperability.

We are currently considering the adoption of modelling features from ORM [5] conceptual modelling methodology and representation. Its adoption would have the advantage of leveraging the vast research which has been carried on supporting the user in the modelling tasks; including the integration of natural language generation. The use of ORM modelling style would require also a redesign of the reasoning tasks in order to align the inferences to the new graphical representation.

On the interface we are improving the automatic layout algorithms and working on the support of undo actions. We also plan to include a role browser tab to show the role hierarchy in the same style of the class browser. Moreover, we are improving the interoperability with other tools by tackling the import and export compatibility with XMI and OWL. 


\section{Bibliography}

[1] S. Bechhofer, I. Horrocks, C. Goble, and G. Stevens. Oiled: a reason-able ontology editor for the semantic web. In Proceedings of KI2001, Joint German/Austrian conference on Artificial Intelligence, pages 396-408. Springer-Verlag, 2001.

[2] S. Bechhofer, R. Möller, and P. Crowther. The dig description logic interface. In Proceedings of the 2003 International Workshop on Description Logics (DL2003), volume 81 of CEUR Workshop Proceedings. CEUR-WS.org, 2003.

[3] P. Fillottrani, E. Franconi, and S. Tessaris. The new icom ontology editor. In Proceedings of the 2006 International Workshop on Description Logics (DL2006), volume 189 of CEUR Workshop Proceedings. CEUR-WS.org, 2006.

[4] E. Franconi and G. Ng. The i.com tool for intelligent conceptual modeling. In Proceedings of the 7th International Workshop on Knowledge Representation meets Databases (KRDB 2000), volume 29 of CEUR Workshop Proceedings. CEURWS.org, 2000.

[5] T.A. Halpin, A.J. Morgan, and T. Morgan. Information modeling and relational databases. Morgan Kaufmann series in data management systems. Elsevier/Morgan Kaufman Publishers, 2008.

[6] M. Horridge, S. Bechhofer, and O. Noppens. Igniting the owl 1.1 touch paper: The owl api. In Proceedings of the OWLED 2007 Workshop on OWL: Experiences and Directions, volume 258 of CEUR Workshop Proceedings. CEUR-WS.org, 2007.

[7] M. Jarke, C. Quix, D. Calvanese, M. Lenzerini, E. Franconi, S. Ligoudistianos, P. Vassiliadis, and Y. Vassiliou. Concept based design of data warehouses: The dwq demonstrators. In Proceedings of the 2000 ACM SIGMOD International Conference on Management of Data, page 591. ACM, 2000.

[8] H. Knublauch, R. W. Fergerson, N. F. Noy, and M. A. Musen. The protégé owl plugin: An open development environment for semantic web applications. In Proceedings of ISWC 2004: Third International Semantic Web Conference, volume 3298 of Lecture Notes in Computer Science. Springer, 2004.

[9] T. Liebig, M. Luther, O. Noppens, M. Rodriguez, D. Calvanese, M. Wessel, M. Horridge, S. Bechhofer, D. Tsarkov, and E. Sirin. Owllink: Dig for owl 2. In Proceedings of the OWLED 2008 Workshop on OWL: Experiences and Directions, volume 432 of CEUR Workshop Proceedings. CEUR-WS.org, 2008.

[10] C. Lutz, F. Baader, E. Franconi, D. Lembo, R. Möller, R. Rosati, U. Sattler, B. Suntisrivaraporn, and S. Tessaris. Reasoning support for ontology design. In Proceedings of the OWLED 2006 Workshop on OWL: Experiences and Directions, volume 216 of CEUR Workshop Proceedings. CEUR-WS.org, 2006. 